\title{
Recurrent Aplastic Anemia
}

National Cancer Institute

\section{Source}

National Cancer Institute. Recurrent Aplastic Anemia. NCI Thesaurus. Code C153293.

The reemergence of aplastic anemia after a period of remission. 